\title{
Healthy eating attitudes and healthy living: An examination of the relationship between attitudes, food choices and lifestyle behaviours in a representative sample of Irish adults
}

\author{
P. Naughton ${ }^{1}$, S. McCarthy ${ }^{1}$ and M. McCarthy ${ }^{2}$ \\ ${ }^{1}$ Food Market \& Consumer Research Group, Teagasc Food Research Centre, Ashtown, Dublin 15 and \\ ${ }^{2}$ Department of Food Business and Development, University College Cork, Cork, Ireland
}

\begin{abstract}
The most recent national study into the food and nutrient intakes of Irish adults indicates that dietary recommendations do not coincide with actual consumption on a general population level. An individual's compliance with dietary recommendations is likely to be associated with their food choice attitudes. Attitudes are a fundamental component of behavioural motivation and are generally defined in terms of people's overall evaluations (favourable or unfavourable) of performing behaviour ${ }^{(1)}$. It is assumed that individuals who hold positive attitudes are more likely to engage in behaviours that support their attitudes ${ }^{(2)}$. For example, a person who has strong positive healthy eating attitude would be expected to consume more fruit and vegetables than a person with a weak attitude. The objective of this study is to compare people's dietary behaviours with their attitudes towards healthy eating while accounting for dietary related lifestyle factors.

The National Adult Nutrition Survey (NANS) is a cross-sectional study into the nutrient intakes of Irish adults aged 18 years and over. The survey was conducted in the Republic of Ireland during 2008-2011 on a representative sample of 1500 adults (740 males, 760 females). Food and beverage intake data was collected using a four day diary. Information related to people's food choice attitudes, physical activity and socio-demographics was collected concurrently using self-completion questionnaires. Through exploratory factor analysis a healthy eating attitude scale comprising seven statements was identified and internal consistency of the scale was verified by calculating Cronbach's alpha $(\alpha=0.808)$. On average people were positively disposed to eating a healthy diet $(\bar{X}=5.34$ (out of 7$)$, $\mathrm{SD}=0.93)$. Using the $33^{\text {rd }}$ and $66^{\text {th }}$ percentile points as cut-offs a dichotomous variable comprising strong healthy eating attitudes $(\bar{X}=6.33, \mathrm{SD}=0.93)$ and weak healthy eating attitudes $(\bar{X}=4.37, \mathrm{SD}=0.93)$ was estimated. Logistic regression analysis was used to determine whether socio-demographic, consumption and lifestyle variables are associated with having a strong healthy eating attitude. A combination of Pearson's correlation analysis and the variance inflation factor (VIF) indicated that multicollinearity was not occurring in the data.

The findings showed that having a healthy eating attitude was significantly and positively related to healthy eating and healthy lifestyle behaviours. Women, increasing age, normal BMI, regular exercise and increasing intakes of fruit and vegetables were associated with a higher odds ratio (OR) for having a strong healthy eating attitude. An increased intake of high calorie foods and increased time spent watching T.V. was associated with a decreased OR for positive attitudes towards healthy eating. In conclusion, healthy eating promotions directed at women and older adults should focus on supporting people's motivations to attain a healthy diet by addressing issues such as dietary self-efficacy and unhealthy eating habits. For men and younger adults, healthy eating promotions will need to address the issues underlying their weak attitudes towards healthy eating.
\end{abstract}

1. Ajzen \& Fishbein (2000) Euro Review Soc Psychol 84, 888-918.

2. Eagly and Chaiken (1993) The Psychology of Attitudes. Fort Worth: Harcourt Brace Jovanovich. 